\title{
ChemComm
}

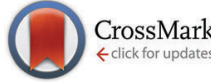

Cite this: Chem. Commun., 2014, 50, 10137

Received 13th April 2014, Accepted 15th July 2014

DOI: $10.1039 / c 4 c c 02723 j$

www.rsc.org/chemcomm

\section{Dynamic nuclear polarisation NMR of nanosized zirconium phosphate polymer fillers $\dagger$}

\author{
Fabio Ziarelli, ${ }^{a}$ Mario Casciola, ${ }^{\mathrm{b}}$ Monica Pica, ${ }^{\mathrm{b}}$ Anna Donnadio, ${ }^{\mathrm{b}}$ Fabien Aussenac, ${ }^{\mathrm{c}}$ \\ Claire Sauvée, $^{d}$ Donatella Capitani*e ${ }^{*}$ and Stéphane Viel ${ }^{* d}$
}

\begin{abstract}
Surface functionalisation with organic modifiers of multi-layered zirconium phosphate (ZrP) nanoparticles used as polymer fillers can be directly probed by dynamic nuclear polarisation NMR, which provides unambiguous evidence of the presence of $\mathrm{P}-\mathrm{O}-\mathrm{C}$ chemical bonds at the surface of the ZrP layers, thereby confirming successful functionalisation.
\end{abstract}

$\alpha$-Type monohydrogen zirconium phosphate (ZrP) particles are made by the packing of planes of zirconium atoms sandwiched between monohydrogen phosphate groups (hereafter referred to as ZrP layers), which are held together by weak van der Waals interactions. ZrP particles are promising inorganic polymer fillers that can be used either with charged or neutral polymers depending on the chemical properties of the surface of their constituting layers. Precisely, the presence of $\mathrm{POH}$ groups on the surface of the layers favours the use of $\mathrm{ZrP}$ particles with polymers possessing polar groups (e.g. starch) ${ }^{1}$ whereas the surface of the layers must be organically functionalised when using $\mathrm{ZrP}$ particles with neutral polymers, so as to promote stronger interactions with the polymer matrix. ${ }^{2,3}$ Accurate chemical characterization of the surface of the $\mathrm{ZrP}$ layers is hence tantamount to controlling the ultimate properties of the $\mathrm{ZrP}$ particles, and this is usually achieved indirectly through a combination of techniques, such as infrared spectroscopy, thermogravimetric analysis, and solid-state NMR (SSNMR). In principle, SSNMR alone could provide the required information with almost atomic resolution, but its intrinsically low sensitivity precludes direct analysis of

\footnotetext{
${ }^{a}$ Aix Marseille Université, Centrale Marseille, CNRS,

Fédération des Sciences Chimiques FR 1739, F-13397 Marseille, France

${ }^{b}$ Università degli studi di Perugia, Dipartimento di Chimica-CEMIN,

I-06123 Perugia, Italy

${ }^{c}$ Bruker Biospin SAS, F-67160 Wissembourg, France

${ }^{d}$ Aix Marseille Université, CNRS, ICR UMR 7273, F-13397 Marseille, France.

E-mail:s.viel@univ-amu.fr

${ }^{e}$ Laboratorio NMR “Annalaura Segre”, Istituto di Metodologie Chimiche, CNR, o0015 Monterotondo (Roma), Italy. E-mail: donatella.capitani@cnr.it

$\dagger$ Electronic supplementary information (ESI) available: Detailed experimental protocols. See DOI: $10.1039 / \mathrm{c} 4 \mathrm{cc} 02723 \mathrm{j}$
}

diluted signals, such as those arising from the surface of materials. Therefore, direct (and unambiguous) assessment of surface functionalisation of nanosized ZrP polymer fillers remains highly challenging. Dynamic nuclear polarisation surface enhanced NMR spectroscopy (DNP SENS) ${ }^{4}$ could potentially circumvent this difficulty. DNP enhances the sensitivity of SSNMR experiments through the microwave driven transfer of polarisation of unpaired electrons to nuclei. ${ }^{5-9}$ The particularity of the DNP SENS approach lies in its ability to probe the surface species. By incipiently wetting the solid with a radical containing solution, the polarisation gets transferred upon microwave irradiation from the unpaired electrons of the radicals (the so-called DNP polarising agents) to the nearby protons of the solvent molecules. This enhanced ${ }^{1} \mathrm{H}$ magnetization then spreads out through the medium by spin diffusion and eventually reaches the vicinity of the material surface, where it is transferred to a heteronucleus of interest by cross polarisation techniques. While DNP SENS has already been used in a range of applications, ${ }^{4}$ including for the investigation of nanoparticles, ${ }^{10-12}$ it has never been applied, to the best of our knowledge, for probing the presence of chemical covalent bonds on the surface of nanoparticles.

Herein, DNP SENS was used to characterize nanosized ZrP polymer fillers obtained by reacting ZrP gels with 1,2-epoxydodecane, ${ }^{13}$ which holds promise as a mechanical strengthener for polymer electrolyte membranes. ${ }^{14}$ Specifically, the sensitivity enhancement provided by DNP allowed two-dimensional (2D) ${ }^{31} \mathrm{P}-{ }^{13} \mathrm{C}$ dipolar correlation experiments ${ }^{15}$ at natural ${ }^{13} \mathrm{C}$ abundance ( $\sim 1 \%$ ) to be carried out on diluted $\mathrm{ZrP}$ samples (the amount of alkyl chains grafted onto ZrP nanoparticles was $\sim 3.5 \mu \mathrm{mol} \mathrm{mg}{ }^{-1}$ ), experiments that are unfeasible using conventional SSNMR instrumentation due to lack of sensitivity. Dipolar correlation experiments directly evidence dipolar contacts - hence, spatial proximities - between the nuclei under investigation. Combining these data with the observation of specific chemical shifts in the DNP-enhanced ${ }^{13} \mathrm{C}$ and ${ }^{31} \mathrm{P}$ CPMAS spectra is shown here to provide direct and unambiguous evidence for the presence of $\mathrm{P}-\mathrm{O}-\mathrm{C}$ bonds at the surface of the $\mathrm{ZrP}$ layers. In particular, the $\mathrm{ZrP}$ sample functionalised with 1,2-epoxydodecane was 


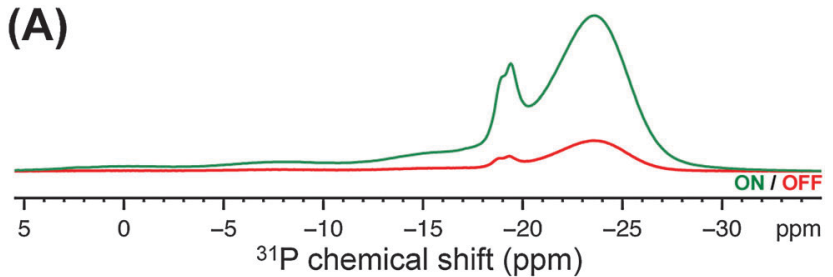

(B)

prepared by incipient wetness impregnation ${ }^{16}$ (see ESI $\dagger$ ) using a $14 \mathrm{mM}$ aqueous solution of a recently described dinitroxide (i.e. PyPOL). ${ }^{17}$ Dinitroxides are currently the most efficient DNP polarizing agents for high-resolution DNP SSNMR experiments. ${ }^{17,18}$ The first indication of the sensitivity gain afforded by DNP is illustrated in Fig. 1, where the DNP-enhanced ${ }^{31} \mathrm{P}$ and ${ }^{13} \mathrm{C}$ CPMAS spectra of the functionalised $\mathrm{ZrP}$ sample are reported. Comparing the signal intensities of the spectra obtained with the microwave field on $\left(I_{\mathrm{ON}}\right)$ and off $\left(I_{\mathrm{OFF}}\right)$, led to a sensitivity increase $\left(\varepsilon_{\mathrm{X}, \mathrm{CP}}=I_{\mathrm{ON}} / I_{\mathrm{OFF}}\right.$, where $\mathrm{X}$ is ${ }^{31} \mathrm{P}$ or $\left.{ }^{13} \mathrm{C}\right)$ of 6 and 8 , respectively $\neq$ Precisely, upon comparing the signal-to-noise ratio $(\mathrm{S} / \mathrm{N})$ per unit of time of the DNP SSNMR experiment with the $\mathrm{S} / \mathrm{N}$ per unit of time of the SSNMR experiment without DNP (at room temperature), the so-called absolute sensitivity ratio (ASR), ${ }^{19,20}$ an ASR value of 10 was obtained, which translated into a time saving factor of 100 . This substantial sensitivity enhancement allowed us to perform the ${ }^{31} \mathrm{P}-{ }^{13} \mathrm{C}$ SSNMR dipolar correlation experiment shown in Fig. 2 within a realistic experimental time frame ( $\sim 2$ days), while the same experiment would have prohibitively required more than 200 days on comparable SSNMR instrumentation without DNP and at room temperature.

The reaction between 1,2-epoxydodecane and the $\mathrm{POH}$ groups of crystalline $\mathrm{ZrP}$ may lead to the formation of 2 types of functional groups (Forms a and $\mathrm{b}$ in the inset of Fig. 2A). ${ }^{13}$ For ZrP nanoparticles, spectral deconvolution of the DNP-enhanced ${ }^{31} \mathrm{P}$ CPMAS spectrum (Fig. 1A) confirmed the presence of two main forms in an approximate $2: 1$ ratio (at -23 and $-24 \mathrm{ppm}$, respectively), and revealed the presence of a few minor ${ }^{31} \mathrm{P}$ resonances due to the unreacted species (see $\mathrm{ESI} \dagger$ ). Based on the assignment of organically modified crystalline $\mathrm{ZrP},{ }^{13}$ these ${ }^{31} \mathrm{P}$ resonances could be attributed to Forms a and b, respectively. Most importantly, the correlation between $\delta\left({ }^{31} \mathrm{P}\right)-23 \mathrm{ppm}$ and $\delta\left({ }^{13} \mathrm{C}\right) 71 \mathrm{ppm}$ (Fig. 2A), and the correlation between $\delta\left({ }^{31} \mathrm{P}\right)-24 \mathrm{ppm}$ and $\delta\left({ }^{13} \mathrm{C}\right) 79 \mathrm{ppm}$ (Fig. 2B), were clear indications of the presence of covalent $\mathrm{P}-\mathrm{O}-\mathrm{C}$ bonds, thereby unambiguously proving the successful grafting of the alkyl chains onto the surface of the ZrP layers of the nanoparticles. Moreover, because the dipolar interaction falls off very quickly with the internuclear distance, correlations involving shorter internuclear distances are typically predominant in dipolar correlation experiments. As a result, these correlations must be related to the ${ }^{13} \mathrm{C}$ nuclei that are closest to the ${ }^{31} \mathrm{P}$ nuclei, which allowed us to assign the ${ }^{13} \mathrm{C}$ chemical shifts at 71 and $79 \mathrm{ppm}$ to carbons $1 \mathrm{a}$ and $2 \mathrm{~b}$, respectively. This partial assignment was confirmed and completed by the ${ }^{1} \mathrm{H}-{ }^{13} \mathrm{C}$ HETCOR experiment reported in Fig. 3 (recorded without DNP because sensitivity was not an issue in this case). The values of ${ }^{1} \mathrm{H}$ chemical shifts observed in this spectrum allowed us to confirm the above assignment and to assign the last two ${ }^{13} \mathrm{C}$ signals at $71 \mathrm{ppm}$ (due to a $\mathrm{CH}$ group, hence carbon $2 \mathrm{a}$ ) and at $65 \mathrm{ppm}$ (due to a $\mathrm{CH}_{2}$ group, hence carbon $1 \mathrm{~b}$ ). Overall, the resulting assignment was found to be in full agreement with

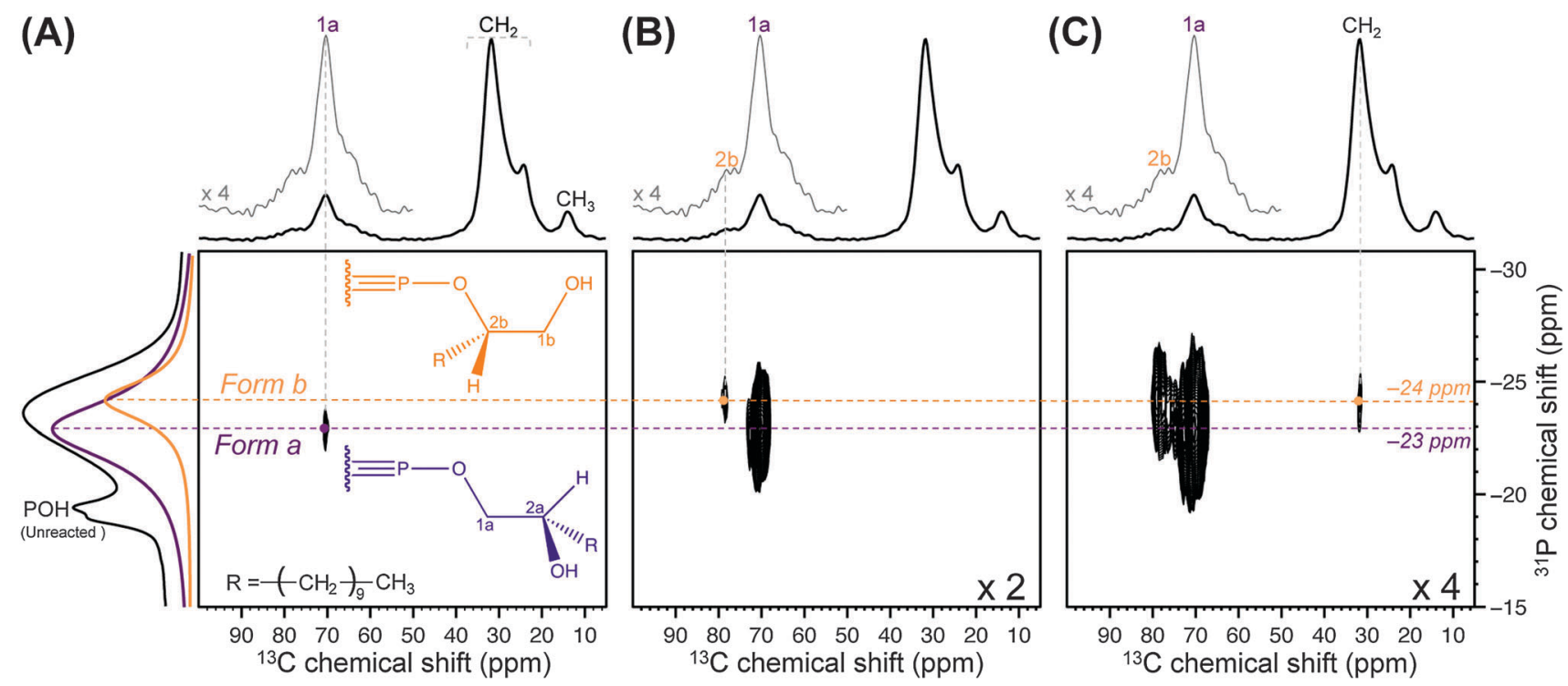

Fig. 2 (A-C) DNP-enhanced 2D ${ }^{31} \mathrm{P}-{ }^{13} \mathrm{C}$ dipolar correlation spectra obtained for the functionalised ZrP sample at natural ${ }^{13} \mathrm{C}$ abundance. With respect to $(A)$, the vertical scale in (B) and (C) has been increased by a factor of 2 and 4 , respectively. 


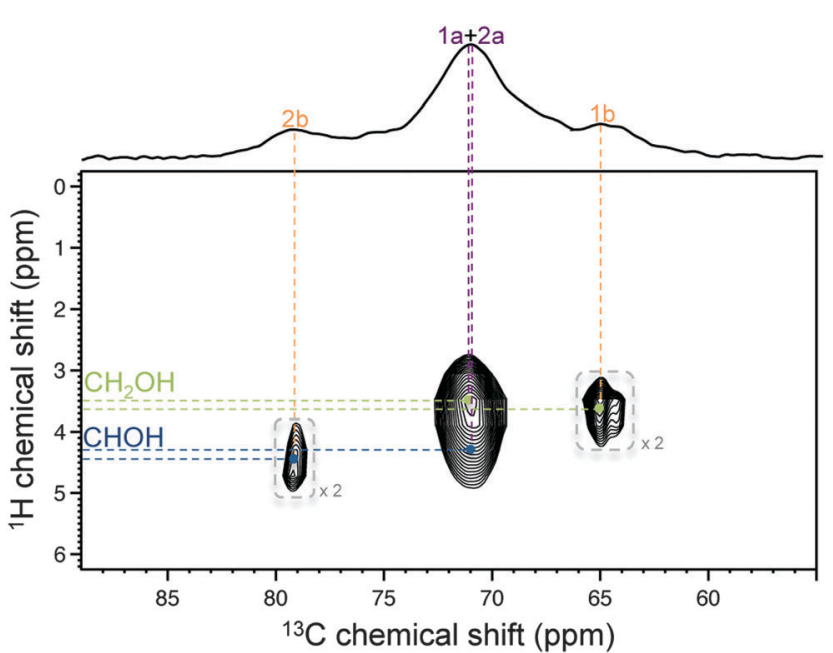

Fig. $3{ }^{1} \mathrm{H}-{ }^{13} \mathrm{C}$ HETCOR spectrum of the functionalised ZrP sample.

the spectral deconvolution of the 50-100 ppm region of the DNPenhanced ${ }^{13} \mathrm{C}$ CPMAS spectrum shown in Fig. 1B (see ESI $\dagger$ ). Finally, in Fig. 2C, another correlation involving Form b at $\delta\left({ }^{13} \mathrm{C}\right)$ $31 \mathrm{ppm}$ could be detected, which was due to the ${ }^{13} \mathrm{C}$ signal of the first $\mathrm{CH}_{2}$ group of the alkyl chain. This correlation was not observed for Form a. This is consistent with the fact that the distance between this $\mathrm{CH}_{2}$ group and the ${ }^{31} \mathrm{P}$ nuclei of the $\mathrm{P}-\mathrm{O}-\mathrm{C}$ bonds (at the surface of the ZrP layers) is shorter in Form $b$ than in Form a. More importantly, no other ${ }^{31} \mathrm{P}-{ }^{13} \mathrm{C}$ dipolar correlation involving the ${ }^{13} \mathrm{C}$ nuclei of the alkyl chains could be observed. This suggested that the alkyl chains were not lying on the surface of the ZrP layers but exhibited an extended conformation that spreads away from the surface of the layers. This point is critical because it indicates that the grafting has potentially rendered the (originally polar) surface of the $\mathrm{ZrP}$ layers more hydrophobic, a necessary prerequisite when using ZrP nanoparticles as fillers for aliphatic polymers.

In summary, DNP allows the surface of polymer fillers to be characterized, and hence appears to be highly relevant for studying the filler/polymer interface in polymeric nanocomposites. This technique is clearly not limited to $\mathrm{ZrP}$ nanoparticles and should be useful for characterizing the surface functionalisation of other types of phosphate materials.

This work has been carried out thanks to the support of the $A^{*}$ MIDEX project (no. ANR-11-IDEX-0001-02) funded by the "Investissements d'Avenir" French Government program, managed by the French National Research Agency (ANR).

\section{Notes and references}

$\ddagger$ These values are substantially lower than those expected with this DNP polarising agent, ${ }^{17}$ which could be related to the possibly inhomogeneous glass formed upon cooling the sample at $\sim 100 \mathrm{~K}$ (presence of water crystals in this case) and/or to a nonuniform dispersion of the radicals in the sample, which are both deleterious to an efficient DNP transfer. In fact, ${ }^{1} \mathrm{H}$ relaxation data reported in the SI suggest that biradicals were not homogeneously dispersed in the sample.

$\S$ This sensitivity enhancement might even be further increased using alternative double cross-polarisation ${ }^{21}$ and/or detection strategies. ${ }^{22}$

1 M. Pica, A. Donnadio, V. Bianchi, S. Fop and M. Casciola, Carbohydr. Polym., 2013, 97, 210-216.

2 L. Y. Sun, W. J. Boo, D. H. Sun, A. Clearfield and H. J. Sue, Chem. Mater., 2007, 19, 1749-1754.

3 B. M. Mosby, A. Diaz, V. Bakhmutov and A. Clearfield, ACS Appl. Mater. Interfaces, 2014, 6, 585-592.

4 A. J. Rossini, A. Zagdoun, M. Lelli, A. Lesage, C. Copéret and L. Emsley, Acc. Chem. Res., 2013, 46, 1942-1951.

5 A. W. Overhauser, Phys. Rev., 1953, 92, 411-416.

6 T. Carver and C. P. Slichter, Phys. Rev., 1953, 92, 212-213.

7 L. R. Becerra, G. J. Gerfen, R. J. Temkin, D. J. Singel and R. G. Griffin, Phys. Rev. Lett., 1993, 71, 3561-3564.

8 D. A. Hall, D. C. Maus, G. J. Gerfen, S. J. Inati, L. R. Becerra, F. W. Dahlquist and R. G. Griffin, Science, 1997, 276, 930-932.

9 Q. Z. Ni, E. Daviso, T. V. Can, E. Markhasin, S. K. Jawla, T. M. Swager, R. J. Temkin, J. Herzfeld and R. G. Griffin, Acc. Chem. Res., 2013, 46, 1933-1941.

10 L. Protesescu, A. J. Rossini, D. Kriegner, M. Valla, A. de Kergommeaux, M. Walter, K. V. Kravchyk, M. Nachtegaal, J. Stangl, B. Malaman, P. Reiss, A. Lesage, L. Emsley, C. Copéret and M. V. Kovalenko, ACS Nano, 2014, 8, 2639-2648.

11 O. Lafon, A. S. L. Thankamony, M. Rosay, F. Aussenac, X. Y. Lu, J. Trebosc, V. Bout-Roumazeilles, H. Vezin and J. P. Amoureux, Chem. Commun., 2013, 49, 2864-2866.

12 U. Akbey, B. Altin, A. Linden, S. Ozcelik, M. Gradzielski and H. Oschkinat, Phys. Chem. Chem. Phys., 2013, 15, 20706-20716.

13 M. Casciola, D. Capitani, A. Donnadio, G. Munari and M. Pica, Inorg. Chem., 2010, 49, 3329-3336.

14 A. Donnadio, M. Pica, D. Capitani and M. Casciola, J. Membr. Sci., 2014, 462, 42-49.

15 J. Schaefer, R. A. McKay and E. O. Stejskal, J. Magn. Reson., 1979, 34, 443-447. 16 A. Lesage, M. Lelli, D. Gajan, M. A. Caporini, V. Vitzthum, P. Mieville, J. Alauzun, A. Roussey, C. Thieuleux, A. Mehdi, G. Bodenhausen, C. Coperet and L. Emsley, J. Am. Chem. Soc., 2010, 132, 15459-15461.

17 C. Sauvée, M. Rosay, G. Casano, F. Aussenac, R. T. Weber, O. Ouari and P. Tordo, Angew. Chem., Int. Ed., 2013, 52, 10858-10861.

18 A. Zagdoun, G. Casano, O. Ouari, M. Schwarzwälder, A. J. Rossini, F. Aussenac, M. Yulikov, G. Jeschke, C. Copéret, A. Lesage, P. Tordo and L. Emsley, J. Am. Chem. Soc., 2013, 135, 12790-12797.

19 H. Takahashi, D. Lee, L. Dubois, M. Bardet, S. Hediger and G. De Paëpe, Angew. Chem., Int. Ed., 2012, 51, 11769.

20 H. Takahashi, B. Viverge, D. Lee, P. Rannou and G. De Paëpe, Angew. Chem., Int. Ed., 2013, 52, 6979-6982.

21 S. C. Christiansen, N. Hedin, J. D. Epping, M. T. Janicke, Y. del Amo, M. Demarest, M. Brzezinski and B. F. Chmelka, Solid State Nucl. Magn. Reson., 2006, 29, 170-182.

22 T. Kobayashi, O. Lafon, A. S. L. Thankamony, I. I. Slowing, K. Kandel, D. Carnevale, V. Vitzthum, H. Vezin, J. P. Amoureux, G. Bodenhausen and M. Pruski, Phys. Chem. Chem. Phys., 2013, 15, 5553-5562. 